\title{
Signal Optimization for UWB Radio Systems
}

Dong Mei Shan ${ }^{1}$, Zhi Ning Chen ${ }^{2}$, Member, IEEE, Xuan Hui $\mathrm{Wu}^{1,2}$, Student Member, IEEE

${ }^{1}$ Department of Electrical and Computer Engineering

National University of Singapore

10 Kent Ridge Crescent, Singapore 119260

${ }^{2}$ Department of Radio Systems, Institute for Infocomm Research

20 Science Park Road, \#02-21/25 TeleTech Park, Singapore 117674

E-mail: g0301347@nus.edu.sg, chenzn@i2r.a-star.edu.sg, xuanhui_wu@ieee.org

\begin{abstract}
This paper presents and studies three frequency-domain models for optimizing source pulses and detection templates in ultra-wideband radio systems. The optimization aims mainly at maximizing the EIRP band efficiency in the free space and the output of correlation detection at a receiver. These models are based on the Differential Evolution, an improved version of the Genetic Algorithm, and carried out on a set of UWB signals with given mathematical forms. As examples, these models are used to optimize the UWB signals for both narrowband thin-wire and wideband planar antenna systems. In addition, the optimized results are validated by non-optimization simulation.
\end{abstract}




\section{Introduction}

The ultra-wideband (UWB) systems, as a viable candidates for short-range high-speed radio services, have two essential requirements. The first is to alleviate the possible interference with other existing systems. UWB radio systems, which operate with extremely large bandwidths, should coexist with many existing systems [1]. Therefore, the equivalent isotropically radiated power (EIRP) must comply with the regulation by some organizations, for example, the Federal Communications Commission (FCC) in USA. The second requirement is the optimal receiving characteristics, which is to say with high fidelity, at the receiver side. For a correlation receiver used in a UWB radio system, the higher fidelity means the larger energy output of correlation detection.

Based on the requirements and the need to fully utilize the allocated bandwidth, the parameters for a specific kind of base-band pulses at the transmitter side and in the template at the receiver side should be optimized for the maximum EIRP band efficiency and the maximum correlation coefficient. Usually, the optimal results can be obtained by simulation with discrete sample points in parameter space. However, this procedure is time-consuming and inaccurate due to the discretization. Thus, optimization methods are adopted instead. In order to predict the received signals for different source pulses, the transmitting/receiving antenna pair is treated as a linear time invariant (LTI) system, which is defined by a transfer function in the frequency-domain.

This paper presents and investigates three frequency-domain models for optimizing the source pulses and detection templates in UWB radio systems. The optimization is carried out on a set of UWB signals with fixed mathematical forms, and determines the optimal signal parameters. Because of the complexity of the optimization models, the Differential Evolution (DE), an improved version of the Genetic Algorithm (GA) is adopted to obtain the optimal results, 
considering the several advantages of the DE, such as good self adaptation and fast convergence $[2,3]$. These models are used to optimize the UWB signals for a narrowband thin-wire dipole antenna system, and a wideband planar antenna system. Particularly, the optimized results are validated by non-optimization simulation.

\section{Optimization models in the frequency-domain}

Consider a transmitting/receiving antenna system as shown in Figure 1, the optimization models are constructed based on two transfer functions induced in [4]. First, $H_{L G}(\omega)$ stands for the voltage transfer function that relates the output voltage $\left(V_{L}(\omega)\right)$ of the receiving antenna to the source voltage $\left(V_{G}(\omega)\right)$ at the transmitting antenna as given in (1), where $c$ is the speed of light, and $r$ the distance between the transmitting and receiving antennas. Second, $\bar{H}_{E G}(\omega)$ as given in (2) represents a vector transfer function, which relates the radiated electric field $\bar{E}(\omega)$ at an observation point in free space to $V_{G}(\omega)$. Clearly, the transmission time delay is excluded from $H_{L G}(\omega)$ in (1), and $\bar{H}_{E G}(\omega)$ in (2). Although not explicitly shown, it should be understood that $\bar{H}_{E G}(\omega)$ is the function of the elevation and azimuth angles of the transmitting antenna, i.e. $\theta$ and $\phi$, and $H_{L G}(\omega)$ depends on those angles of both the transmitting and receiving antennas.

$$
\begin{aligned}
& V_{L}(\omega)=H_{L G}(\omega) V_{G}(\omega) \exp \left(\frac{-j \omega r}{c}\right) \\
& \bar{E}(\omega)=\bar{H}_{E G}(\omega) V_{G}(\omega) \exp \left(\frac{-j \omega r}{c}\right), \text { where } \bar{H}_{E G}(\omega)=\hat{\theta} H_{E G}^{\theta}(\omega)+\hat{\phi} H_{E G}^{\phi}(\omega)
\end{aligned}
$$

With the transfer functions $H_{L G}(\omega)$ and $\bar{H}_{E G}(\omega), \operatorname{EIRP}(\operatorname{EIRP}(\omega))$, EIRP bandwidth efficiency $(\eta)$, and correlation coefficient $(\mu)$ can be defined in $(3),(4)$, and (5), respectively. In (4), $\mathrm{BW}_{10 \mathrm{~dB}}$ is the $10 \mathrm{~dB}$ bandwidth of EIRP, and $\mathrm{BW}_{\mathrm{UWB}}=10.6-3.1 \mathrm{GHz}=7.5 \mathrm{GHz}$ based on the FCC's indoor emission limits. In $(5), V_{T}(\omega)$ is the template voltage, and $Q_{L}$ as well as $Q_{T}$ are the energy of the received and template signals, respectively. The $V_{L}(\omega)$ without any 
direct current (DC) component, which must be true in our case, guarantees that $0 \leq \mu \leq 1$.

$$
\begin{aligned}
& \operatorname{EIRP}(\omega)=\frac{\left|V_{G}(\omega)\right|^{2}}{120 \pi}\left[\left|H_{E G}^{\theta}(\omega)\right|^{2}+\left|H_{E G}^{\phi}(\omega)\right|^{2}\right] \\
& \eta=\frac{\mathrm{BW}_{10 \mathrm{~dB}}}{\mathrm{BW}_{\mathrm{UWB}}} \\
& \mu=\left|\frac{\int_{0}^{+\infty} V_{L}(t) V_{T}(t) d t}{\sqrt{Q_{L} Q_{T}}}\right|=\left|\frac{\int_{-\infty}^{+\infty} V_{G}(\omega) H_{L G}(\omega) V_{T}^{*}(\omega) d \omega}{\sqrt{\int_{-\infty}^{\infty}\left|V_{G}(\omega) H_{L G}(\omega)\right|^{2} d \omega \int_{-\infty}^{+\infty}\left|V_{T}(\omega)\right|^{2} d \omega}}\right|
\end{aligned}
$$

Generally speaking, the optimization model should involve two parts, the objective function $(\mathrm{Obj})$ and the constraints (cons). Considering the requirements of the UWB systems, the optimization objective is to maximize $\eta$ and $\mu$. The constraint considered here can be described in (6), where $M_{F C C}(\omega)$ stands for the FCC's emission limits mask. In addition, the EIRP's main lobe must be located in the UWB band.

$$
\operatorname{EIRP}(\omega) \leq M_{F C C}(\omega)
$$

Three models listed below are presented to optimize the UWB signals. The optimal $\eta$ and $\mu$ can be obtained separately as in the Model 1, or simultaneously as in the Model 2 and 3.

- Model 1:

- Step 1: $O b j=$ Maximize $\{\eta\}$, subject to cons, to obtain the optimal source pulse.

- Step 2: $O b j=$ Maximize $\{\mu\}$, with the optimal source pulse obtained from Step 1, to obtain the optimal detection template.

- Model 2: $O b j=$ Maximize $\{\mu+\eta\}$, subject to cons, to obtain the optimal source pulse and detection template.

- Model 3: $O b j=$ Maximize $\{\mu \eta\}$, subject to cons, to obtain the optimal source pulse and detection template. 
Before optimization, the mathematical formulas of the UWB signals should be determined. The source pulse is chosen as a monocycle in the form of (7) with $n=1$, or a modulated Gaussian impulse in the form of (8). The detection template could be a high order derivative Gaussian impulse in the form of (7) with $n \geq 1$, or a truncated cosine signal in the form of $(9)$, where $\mathrm{W}(t)$ is a unit rectangular function whose duration and position on time scale are adaptively determined by the received signal $\left(V_{L}(t)\right)$. The $\tau_{1}$ and $\tau_{2}$ in the definition of $\mathrm{W}(t)$ are the first and last time spots where the absolute value of $V_{L}(t)$ drops to be $10 \%$ of its peak value, taking the time spot corresponding to the peak value as the reference on time. The relationship between the $\mathrm{W}(t)$ and $V_{L}(t)$ is illustrated in Figure 2, where $A$ is the peak value of $V_{L}(t)$.

$$
\begin{aligned}
& g_{n}(t)=\frac{d^{n}}{d t^{n}} \exp \left[-\left(\frac{t}{\sigma}\right)^{2}\right] \\
& g_{\sin }(t)=\sin \left(\omega_{c} t\right) \exp \left[-\left(\frac{t}{\sigma}\right)^{2}\right] \\
& g_{\text {truncate }}(t)=\cos \left(\omega_{c} t\right) \mathrm{W}(t), \text { where } \mathrm{W}(t)= \begin{cases}1, & \tau_{1} \leq t \leq \tau_{2} \\
0 & , \text { else }\end{cases}
\end{aligned}
$$

The decision variable of all the models mentioned above are the parameters existing in $V_{G}(\omega)$ and $V_{T}(\omega)$. The signal characteristics bring about two uncertainties for the optimization. The first is that it is hard to predict the convex property of $V_{G}(\omega)$ and $V_{T}(\omega)$. The second is that all the parameters may take either discrete value, $n$ in (7), or continuous ones, $\sigma$ in (7) and (8), and $\omega_{c}$ in (8) and (9). Thus, it is difficult to define the types of these optimization models in a common sense. To solve this problem, one method is to design particular algorithm for each specific pair of $V_{G}(\omega)$ and $V_{T}(\omega)$. Another one is to employ the global optimization method. In this paper, regarding the complexity of models, which are always non-convex mixed integer problems, the Differential Evolution (DE), an improved version of the Genetic Algorithm (GA) is employed to obtain the optimal results. 
Like all other evolutionary optimization algorithms, the DE works with a population of solutions. The population $P$ of generation $G$ contains $M$ solution vectors, called individuals of the population. Each vector represents a potential solution for the optimization problem. So the population of generation $G$ contains $M$ individuals, each of which contains $N$ parameters (chromosomes of individuals):

$$
P^{(G)}=\left\{X_{i}^{(G)}\right\}=\left\{x_{i, j}^{(G)}\right\}, \quad i=1, \ldots, M ; j=1, \ldots, N ; G=1, \ldots, G_{\max }
$$

where $G_{\max }$ denotes the maximum generation number in the DE method. Based on the boundaries of the problem variables, the DE method initializes the population $P^{(0)}$ (initial population) at first. After the initialization, the new populations are constructed continuously by three main steps, i.e. mutation, crossover and selection, until the stopping criterion is satisfied $[5,6]$. Currently, there are several variations of the DE method. The particular variation used throughout this investigation is the $\mathrm{DE} / \mathrm{rand} / 1$ scheme [7].

\section{Optimized results}

Based on the study of the design considerations for the source pulses and antennas, the above three models are implemented to optimize the UWB signals for two antenna systems [8]. One is a narrowband system comprising two thin-wire antennas, and the other one is a wideband system comprising two planar antennas. For each system, the transfer functions including $\bar{H}_{E G}(\omega)$ and $H_{L G}(\omega)$ with different antenna orientations are obtained by a commercial EM solver (XFDTD) with an FDTD algorithm. The UWB signals including the source pulse and detection template are optimized for a fixed antenna orientation. Then, with the optimized signals, the antenna system performances for other antenna orientations are investigated. 


\subsection{Thin-wire dipole antenna system}

The antenna orientations are illustrated in Figure 3, where the transmitting and receiving antennas are placed in the same plane, at a distance of $655 \mathrm{~mm}$. The thin-wire antennas are of the same geometry, each with an overall length of $21 \mathrm{~mm}$, and a radius of $0.02 \mathrm{~mm}$. The radiation direction of the transmitting antenna is defined by $\theta$, and the angle of arrival (AOA) of the receiving antenna $\left(\theta^{\prime}\right)$ equals to $90^{\circ}$ in order to achieve the maximum receiving of the electromagnetic energy. Both the internal resistance $\left(R_{0}\right)$ of the voltage source and the load $\left(R_{L}\right)$ of the receiving antenna are $100 \Omega$. The $\left|S_{11}\right|$ and $\left|S_{21}\right|$ in $\mathrm{dB}$ of the antenna system with $\theta=90^{\circ}$ are plotted in Figure 4, where the $\left|S_{11}\right| \leq-10 \mathrm{~dB}$ bandwidth is from $6.52 \mathrm{GHz}$ to 7.18 $\mathrm{GHz}$, and the $-3 \mathrm{~dB}$ bandwidth of $\left|S_{21}\right|$ is from $6.02 \mathrm{GHz}$ to $7.67 \mathrm{GHz}$, locating at the center of the UWB band.

In the optimization of the UWB signals, the source pulse is selected as a monocycle, in the form of (7) with $n=1$, and the detection template selected in the form of (7) with $n \geq 1$, or (9). The optimization is carried out for the antenna orientation with $\theta=\theta^{\prime}=90^{\circ}$, and optimized results are tabulated in Table 1 for the template of (7), and Table 2 for the template of (9). In these two tables, the subscript 1 indicates a parameter for the source pulse, and the subscript 2 for the detection template. Specifically, $\sigma_{1}, \sigma_{2}, f_{2}$, and $n_{2}$ are the time constant of the source pulse, time constant of the detection template, carrier frequency of the detection template, and order of the detection template, respectively. Clearly, the three optimization models demonstrate fairly good and similar results. The similarity in the three sets of results obtained from the three models implies that the incorporation of $\mu$ into $O b j$, both in the Models 2 and 3 , dose not impose much effects on obtaining the optimal source pulse, when compared to that obtained in the first step of Model 1. This may be caused by the little variation in the optimal $\mu$ for different $\eta$. Specially, if no such variation, the Models 2 and 3 becomes two equivalents of Model 1. Consequently, the similar source pulses obtained from the three models 
lead to the similar optimized template pulses. It can also be figured out from the Tables 1 and 2 that the $g_{6}(t)$ template leads to a slightly larger correlation coefficient than the $\cos \left(\omega_{c} t\right) \mathrm{W}(t)$ template. With the optimized source pulse, the received signal can be obtained from (1), and plotted in Figure 5 against the optimized templates.

In order to check whether the optimized results are the real optimal values, simulations without the DE algorithm are carried out in a uniformly discretized parameter space, where each possible combination of the parameters is examined to evaluate the system performances. The optimal results obtained by this non-optimization simulation are compared to those obtained by Model 1 . For different source pulses, the resulting EIRP bandwidth in the $\theta=90^{\circ}$ direction is plotted in Figure 6. The correlation coefficient with different templates are plotted in Figure 7 for the template of (7) with $n$ from 1 to 6 , and Figure 8 for the template of (9). In all the three figures, the optimized results obtained by the DE algorithm well agree with the optimal results.

With the above optimized source pulse and detection templates, the system performances such as the EIRP in other radiation directions, and the pulse detection in other antenna orientations were investigated. The EIRP in the radiation angles of $\theta=10^{\circ}$ to $90^{\circ}$ are plotted against the FCC's indoor emission limits mask in Figure 9, where the EIRP complies with the emission limits in all the directions. For the antenna orientations of $\theta=10^{\circ}$ to $90^{\circ}$ and $\theta^{\prime}=90^{\circ}$, The correlation coefficients with the two optimized templates are plotted in Figure 10. It can be seen that for both the templates, the correlation coefficient with a value around 0.88 keeps stable against $\theta$, implying that the waveform of the received signal changes slightly with the antenna orientation. This is a necessary condition for mobile applications, where the device should work properly with any antenna orientation. In addition, compared to the $\cos \left(\omega_{c} t\right) \mathrm{W}(t)$ template, the $g_{6}(t)$ template is capable of capturing more energy of the received signal for its lager correlation coefficient. With $\theta$ increasing from $10^{\circ}$ to $90^{\circ}$, the difference between the correlation coefficients 
due to the two templates increases from $0.2 \%$ to $1.7 \%$.

\subsection{Planar dipole antenna system}

An antenna system with two identical planar antennas is illustrated in Figure 11(a), where the angles $(\theta, \phi)$ stand for the radiation direction of the transmitting antennas, and $\left(\theta^{\prime}, \phi^{\prime}\right)$ defines the AOA of the receiving antenna. Similar to the thin wire antenna system, the transmitting and receiving antennas are separated at a distance of $655 \mathrm{~mm}$, and $R_{0}=R_{L}=100 \Omega$. The antennas are planar dipoles with four slots located around the feed point, as shown in Figure 11(b). The $\left|S_{11}\right|$ and $\left|S_{21}\right|$ in $\mathrm{dB}$ of the antenna system in the orientation of $\theta=\theta^{\prime}=\phi=\phi^{\prime}=90^{\circ}$ are plotted in Figure 12. Compared to Figure 4, the bandwidth of the planar antenna system is very wide, with the $-10 \mathrm{~dB}$ bandwidth of $\left|S_{11}\right|$ begins from $3.99 \mathrm{GHz}$, and extends beyond 13 GHz. The $-3 \mathrm{~dB}$ bandwidth of $\left|S_{21}\right|$ is from $3.00 \mathrm{GHz}$ to $10.33 \mathrm{GHz}$, covering almost the entire UWB band.

For the face-to-face orientation of $\theta=\theta^{\prime}=\phi=\phi^{\prime}=90^{\circ}$, the three optimization models are applied to optimize the source pulse and detection templates. If the source pulse is selected as a monocycle in the form of (7) with $n=1$, no pulse can make the EIRP comply with the FCC's mask because the monocycle only has one degree of freedom that disables the arbitrary control its central frequency and bandwidth. Therefore, the modulated Gaussian impulse in the form of (8) and with two degrees of freedom is adopted instead. The same template as that for the thin-wire antenna system is selected, either in the form of (7) or (9). The optimized results are tabulated in Table 3 for using the $g_{n}(t)$ template, and Table 4 for the $\cos \left(\omega_{c} t\right) \mathrm{W}(t)$ template. Both the tables show that the three optimization models exhibit almost the same results, due to the same reasons exposed in the discussion of the optimized results for the thinwire dipole antenna system. Compared to the pulse detection in the thin wire antenna system, the two types of templates result in greater difference of about $15 \%$ between the corresponding 
correlation coefficients. The optimized templates are plotted in Figure 13, synchronizing the received signal resulted from the optimized source pulse. It can be seen that the received signal has much shorter time duration and less ringing when compared to that in the thin-wire antenna system due to the broad bandwidth for $\left|S_{21}\right|$. The less dispersion of the signal requires a $g_{n}(t)$ template of lower order.

With the optimized source pulse and detection template, the EIRP in different directions, and the pulse detection in $\theta=90^{\circ}$ and $\phi=90^{\circ}$ planes are investigated. The resulting EIRP due to the optimized source pulse well complies with the FCC's indoor mask in all the directions, as shown in Figure 14. With $\theta^{\prime}=\phi^{\prime}=90^{\circ}$, the correlation coefficient of the pulse detection is plotted in Figure 15 for the $\theta=90^{\circ}$ plane, and Figure 16 for the $\phi=90^{\circ}$ plane. It can be observed that the adoption of $\cos \left(\omega_{c} t\right) \mathrm{W}(t)$ template leads to a relatively stable $\mu$ in both the planes, while the $g_{6}(t)$ only achieves a stable $\mu$ in the $\theta=90^{\circ}$ plane but varies a lot in the $\phi=90^{\circ}$ plane, decreasing from 0.974 to 0.85 with $\theta$ changing from $90^{\circ}$ to $10^{\circ}$.

\section{Conclusions}

The DE method has been successfully applied to optimize the source pulse and detection template of two antenna systems for UWB pulsed radio applications. The optimization objective is to maximize both the EIRP bandwidth efficiency, and the pulse detection performance under the constrains of complying with the FCC's emission limits mask. Three optimization models with different objective functions have been presented, and all of them have obtained fairly good and similar results. The optimized UWB signals have been firstly obtained in an antenna system with face-to-face antenna orientation, and then used to investigate the system performances with other antenna orientations.

As examples, two antenna systems namely the narrowband thin-wire and wideband planar 
antennas, have been considered in this work. Due to the different characteristics of the two antenna systems, the optimized UWB signals for the two systems are greatly different. For the thin-wire antenna system, the optimized source pulse could be a monocycle with its spectrum shaped by the transmitting antenna to meet the FCC's emission limits mask. However, for the wideband planar antenna system, the spectrum shaping function of the transmitting antenna is not so obvious that the monocycle cannot be used to meet the emission limits mask. Therefore, a modulated Gaussian impulse with two degrees of freedom has been adopted instead for the wideband system. For the pulse detection, the $g_{n}(t)$ template results in better detection performance than the $\cos \left(\omega_{c} t\right) \mathrm{W}(t)$ template does. The thin-wire antenna system requires a higher order $g_{n}(t)$ template because its serious dispersion generates more ringing in the received signal. For the planar antenna system, the different selection of the template has led to greatly different stability of the detection performance with antenna orientations. For example, in the $\phi=90^{\circ}$ plane, the correlation coefficient obtained with $g_{3}(t)$ template has changed a lot with $\theta$, while that obtained with $\cos \left(\omega_{c} t\right) \mathrm{W}(t)$ template keeps almost the same. 


\section{References}

[1] R. A. Scholtz, R. Weaver, E. Homier, J. Lee, P. Hilmes, A. Taha, and R. Wilson, 'UWB Radio Deployment Challenges', Proc. IEEE Int. Symp. Personal, Indoor and Mobile Radio Communications, London, U. K., Sep. 2000, vol. 1, pp. 620-625.

[2] Beyer, HoG. G. Deb, 'On the Analysis of Self Adaptive Evolutionary Algorithms', Technical Report No. CI-69/99, May 1999, University of Dornund, Germany.

[3] D. Zaharie, 'On the Explorative Power of Differential Evolution Algorithms', The Third Int. Workshop "Symbolic and Numeric Algorithms on Scientific Computing", Timisoara, Romania, Oct. 2-4, 2001.

[4] D. M. Pozar, 'Waveform Optimizations for Ultrawideband Radio Systems', IEEE Trans. Antennas and Propagat. , 2003, 51, (9), pp. 2335-2345.

[5] A. S. Lieber, C. M. Moles, J. R. Banga, and K. Keller, 'Efficient global optimization of nonconvex and nondifferentialable climate control problems', Projected for submission to Computational Optimization and Applications.

[6] J. Lampinen and I. Zelinka, 'Mixed Variable Non-Linear Optimization by Differential Evolution. In: Zelinka, Ivan (ed.)(1999)', Proceedings of Nostradamus'99, 2nd International Prediction Conference, Zlin, Czech Republic, Oct. 7-8, 1999.

[7] R. Storn and K. Price, 'Differential Evolution - A Simple and Efficient Adaptive Scheme for Global Optimization over Continuous Spaces', Berkeley, CA, Tech. Rep. TR-95-012, 1995.

[8] Z. N. Chen, X. H. Wu, H. F. Li, N. Yang, and M. Y. W. Chia: 'Considerations for Source Pulses and Antennas in UWB Radio Systems', IEEE Trans. Antennas Propagat., 2004, 52, (7). pp. 1739-1748. 


\section{List of Figures}

1 Transmitting/receiving antenna system . . . . . . . . . . . . . . 15

2 Relationship between $\mathrm{W}(t)$ and $V_{L}(t) \ldots \ldots \ldots \ldots \ldots \ldots$

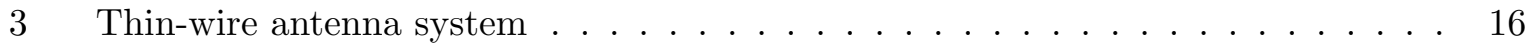

4 S-parameters of the thin-wire antenna system . . . . . . . . . . . 16

$5 \quad$ Pulse detection for the thin wire antenna system $\ldots \ldots \ldots \ldots$

$6 \quad$ EIRP bandwidth efficiency with different monocycles . . . . . . . . . . . 17

$7 \quad$ Pulse detection with the $g_{n}(t)$ template $\ldots \ldots \ldots \ldots \ldots$

8 Pulse detection with the $\cos \left(\omega_{c} t\right) \mathrm{W}(t)$ template $\ldots \ldots \ldots \ldots \ldots$

$9 \quad$ EIRP from the thin-wire antenna . . . . . . . . . . . . . . . . . . 19

10 Correlation coefficient of the thin-wire antenna system with different antenna

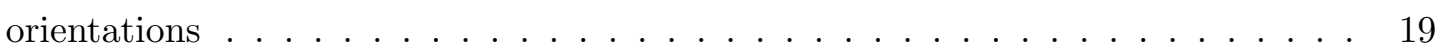

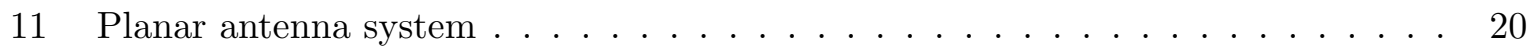

12 S-parameters of the planar antenna system f . . . . . . . . . . . . . 21

13 Pulse detection for the planar antenna system . . . . . . . . . . . . . . 21

14 EIRP from the planar antenna . . . . . . . . . . . . . . . . . 22

15 Correlation coefficient of the planar antenna system in the $\theta=90^{\circ}$ plane . . . . 22

16 Correlation coefficient of the planar antenna system in the $\phi=90^{\circ}$ plane . . . . 23 


\section{List of Tables}

1 Optimized results with the $g_{n}(t)$ template for the thin wire antenna system . . 24

2 Optimized results with the $\cos \left(\omega_{c} t\right) \mathrm{W}(t)$ template for the thin wire antenna system 24

$3 \quad$ Optimized results with the $g_{n}(t)$ template for the planar antenna system . . . . . 24

$4 \quad$ Optimized results with the $\cos \left(\omega_{c} t\right) \mathrm{W}(t)$ template for the planar antenna system 24 

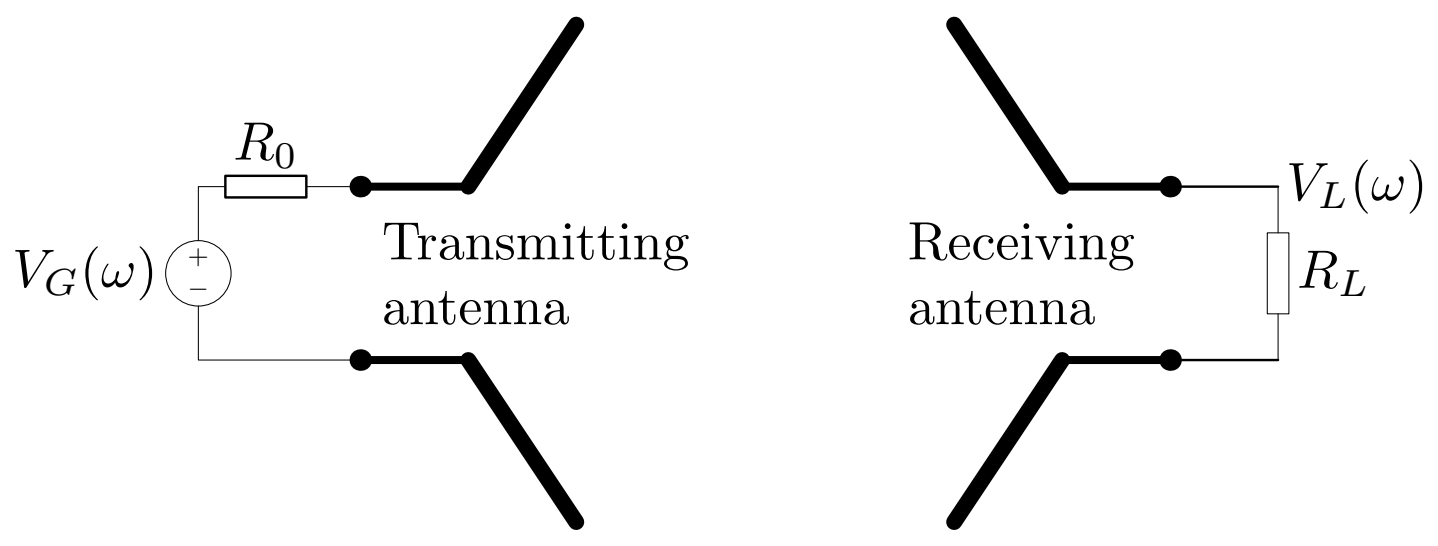

Figure 1: Transmitting/receiving antenna system

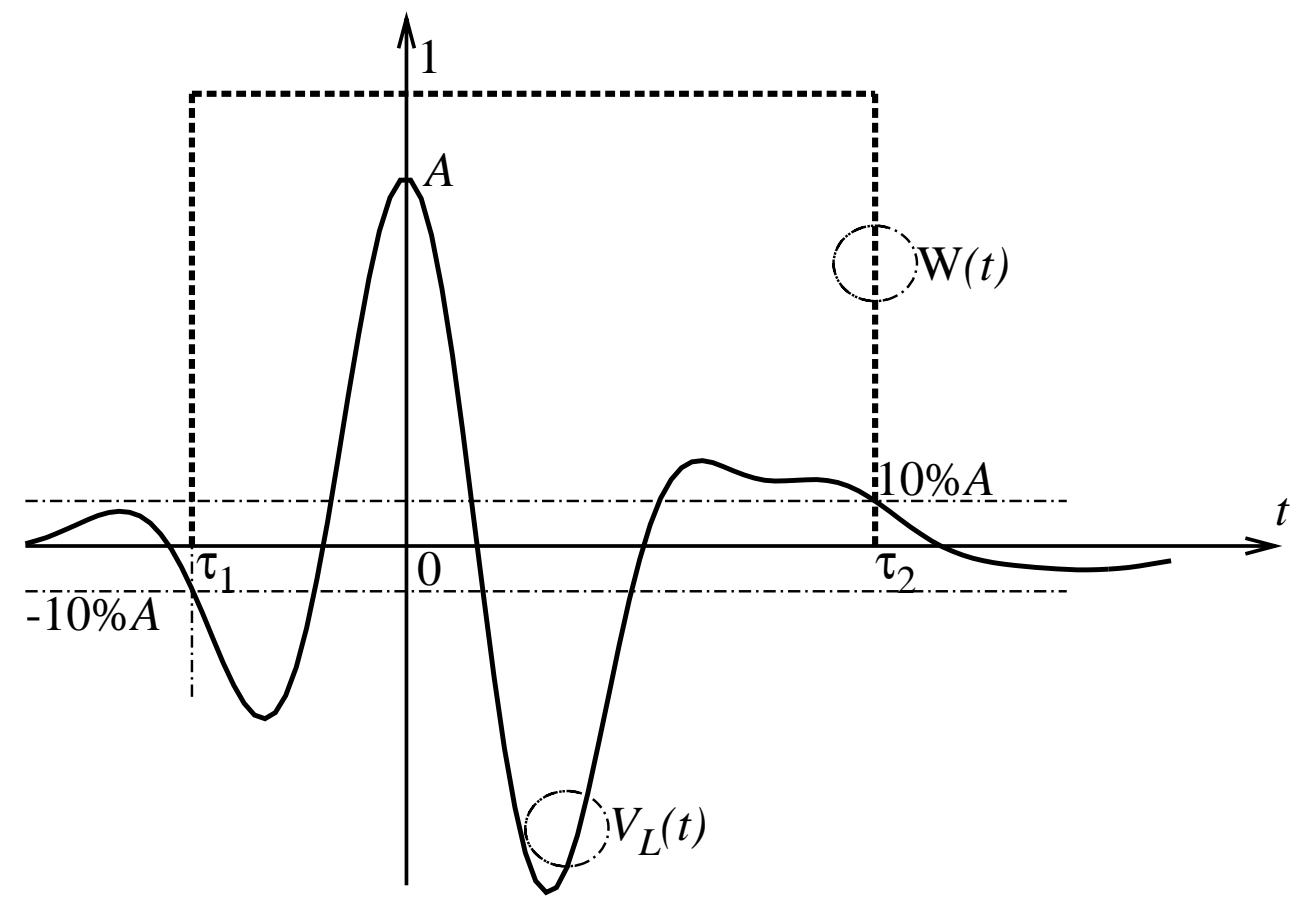

Figure 2: Relationship between $\mathrm{W}(t)$ and $V_{L}(t)$ 


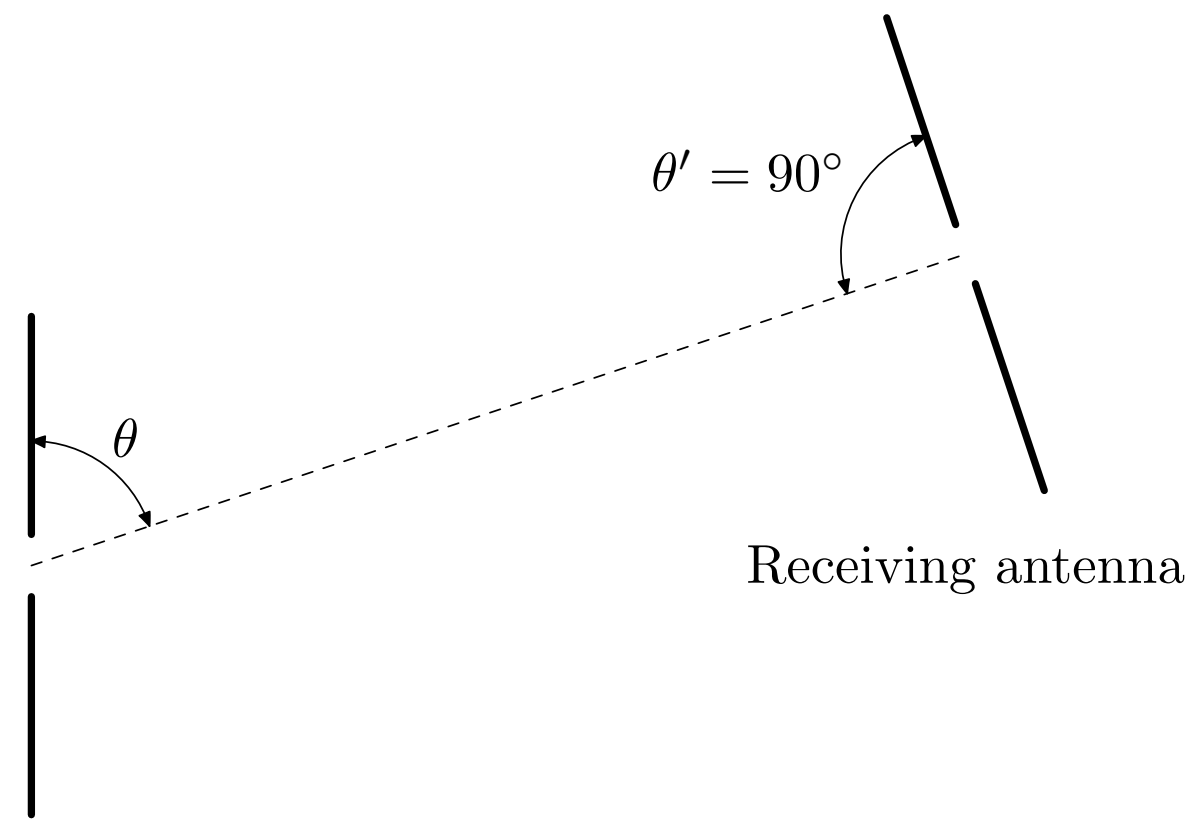

Transmitting antenna

Figure 3: Thin-wire antenna system

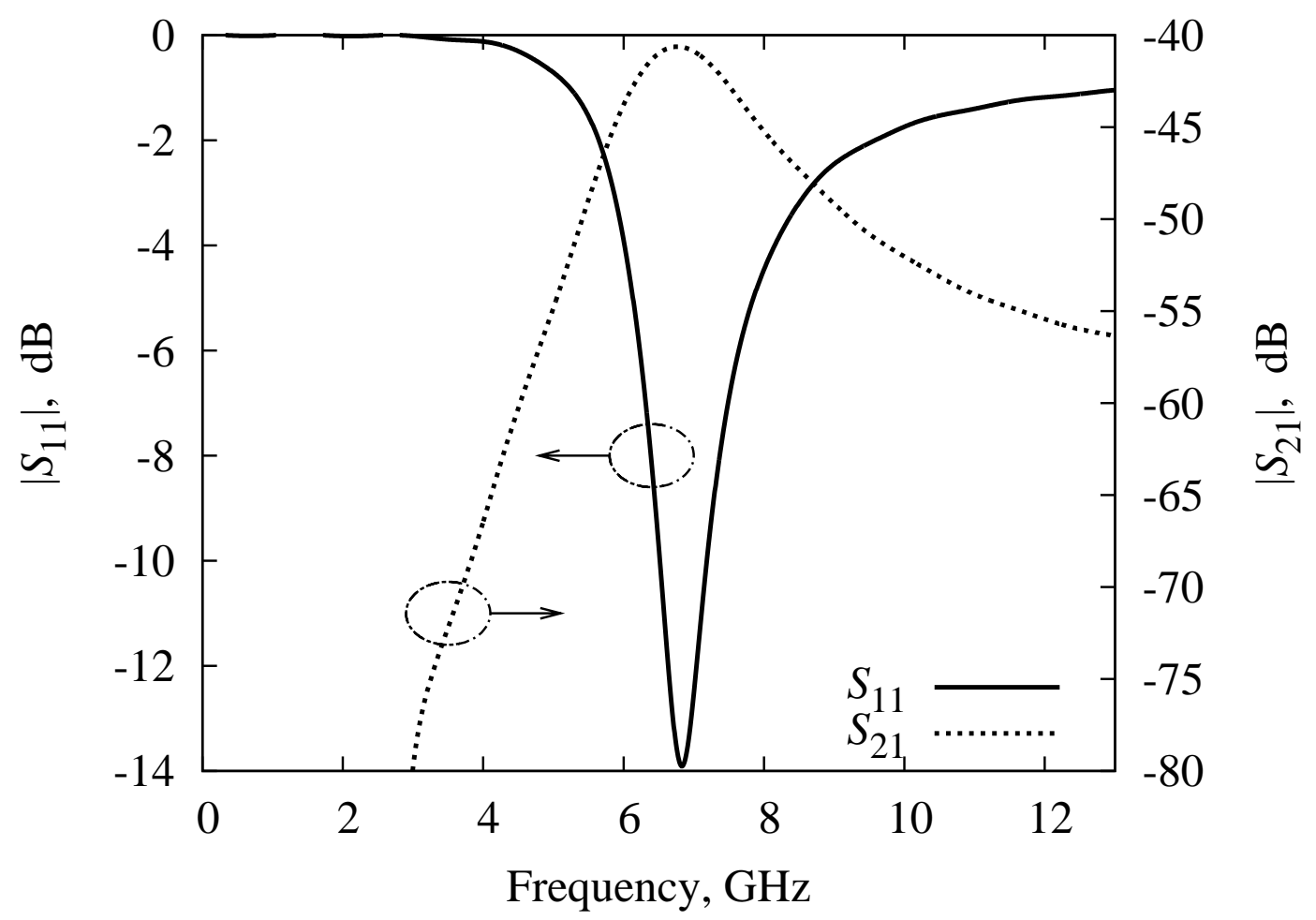

Figure 4: S-parameters of the thin-wire antenna system 


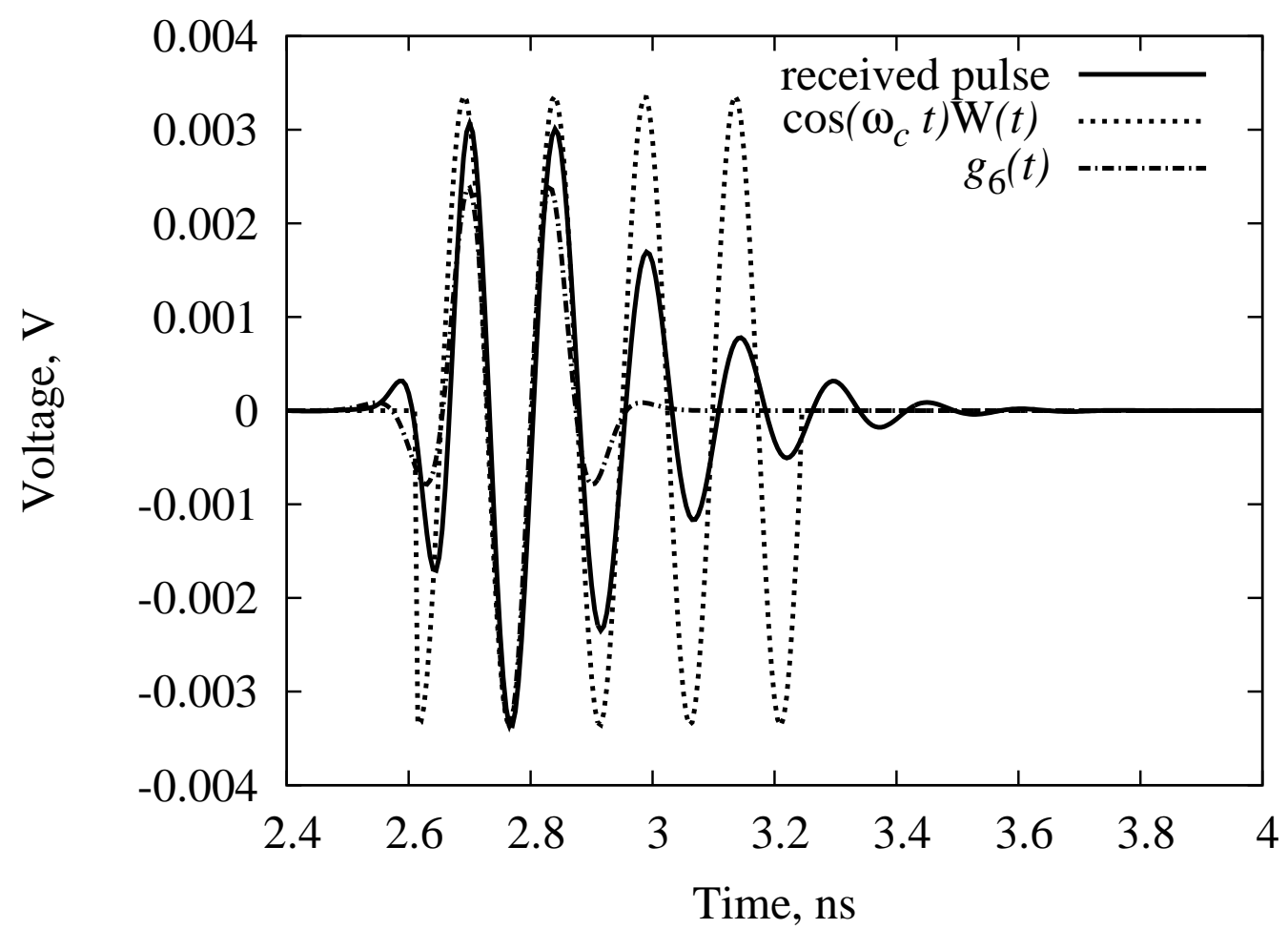

Figure 5: Pulse detection for the thin wire antenna system

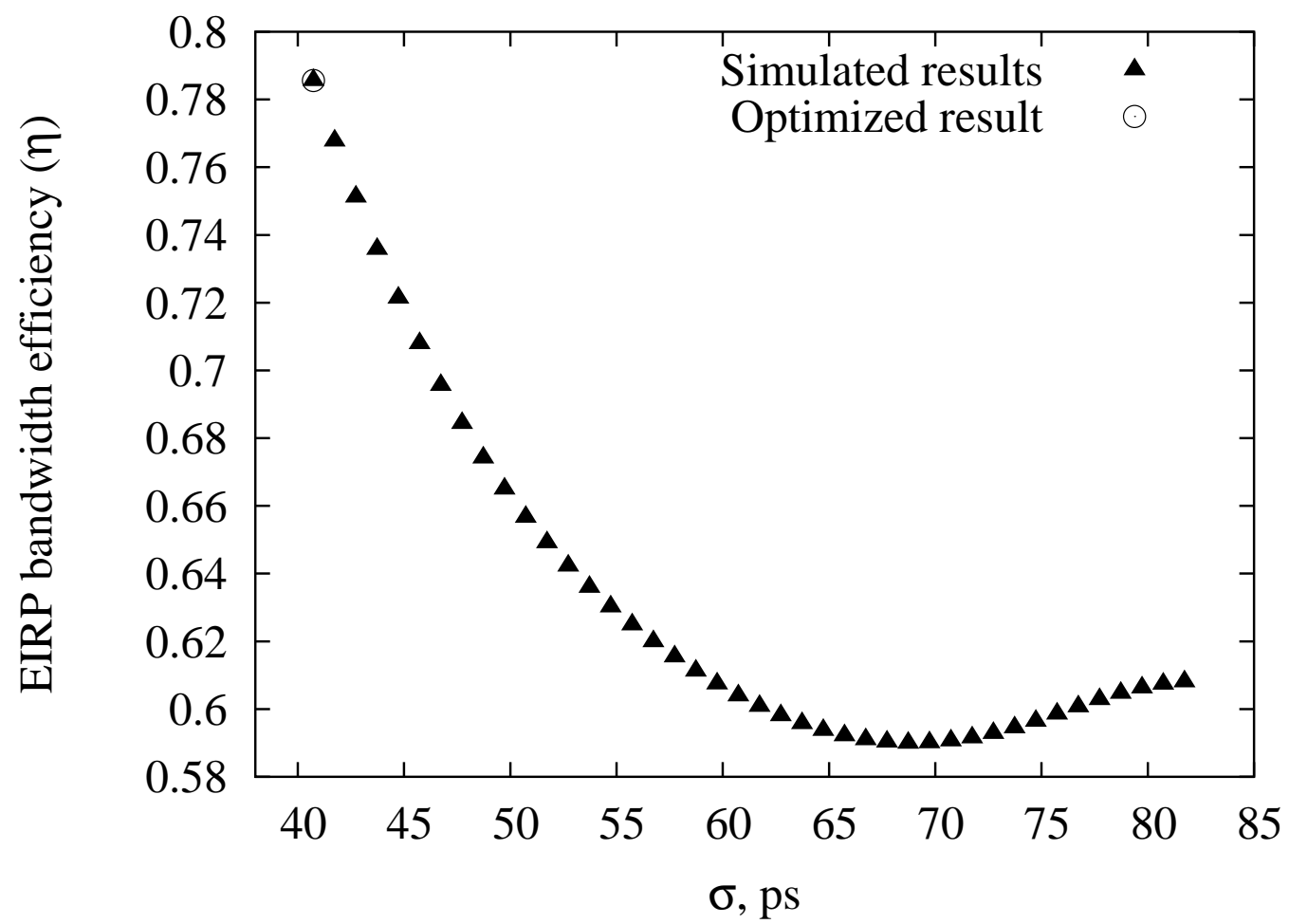

Figure 6: EIRP bandwidth efficiency with different monocycles 


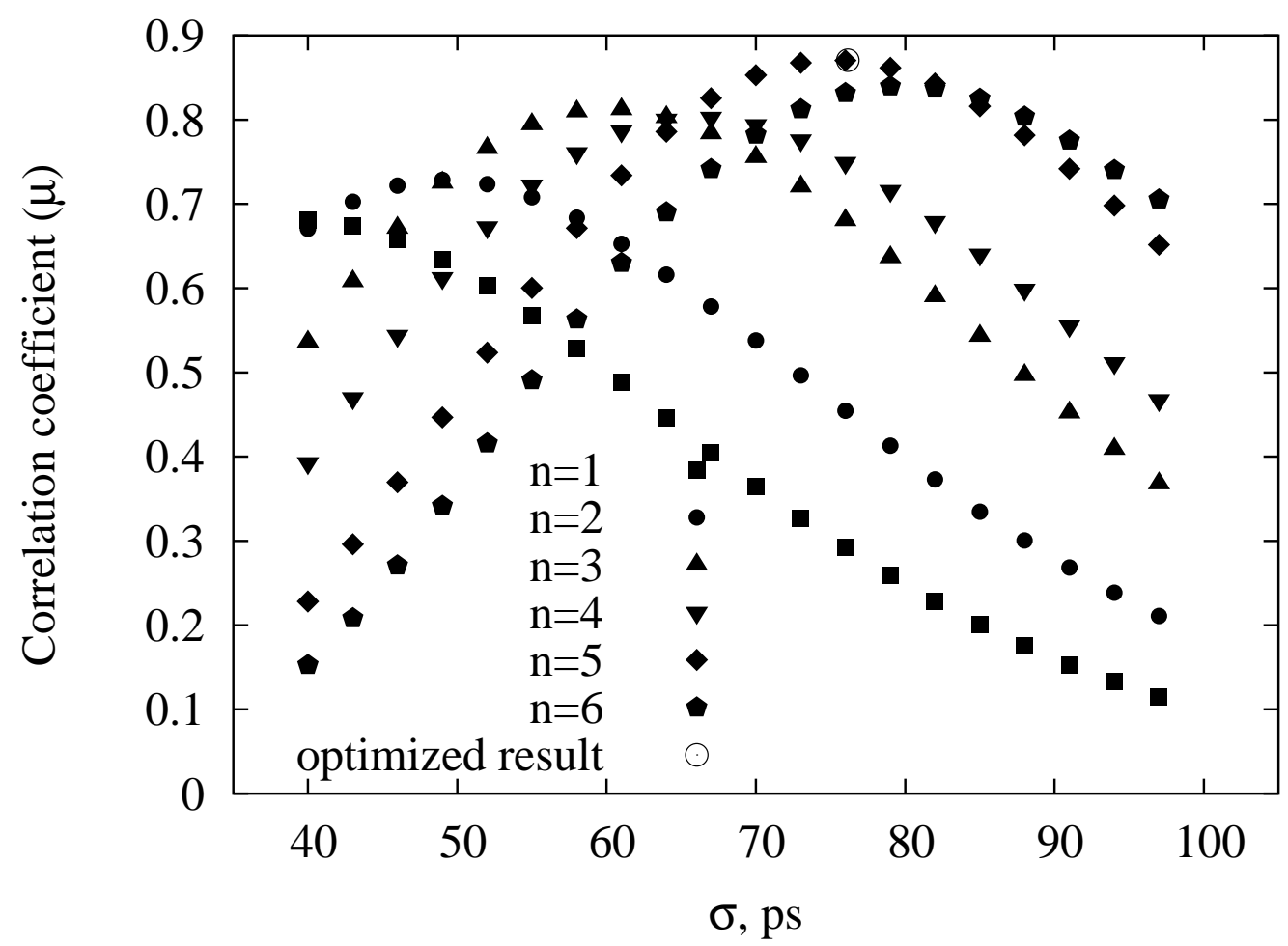

Figure 7: Pulse detection with the $g_{n}(t)$ template

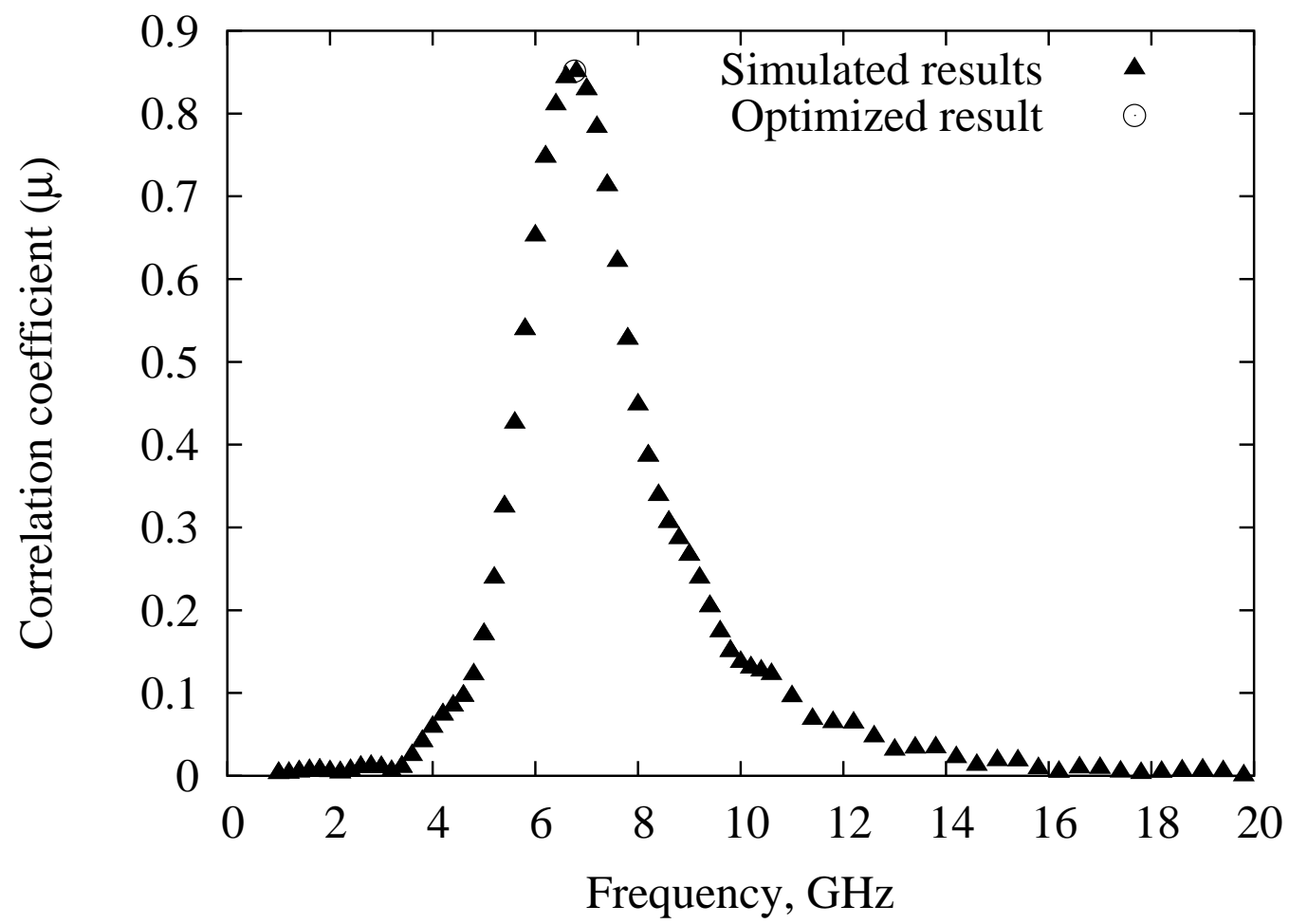

Figure 8: Pulse detection with the $\cos \left(\omega_{c} t\right) \mathrm{W}(t)$ template 


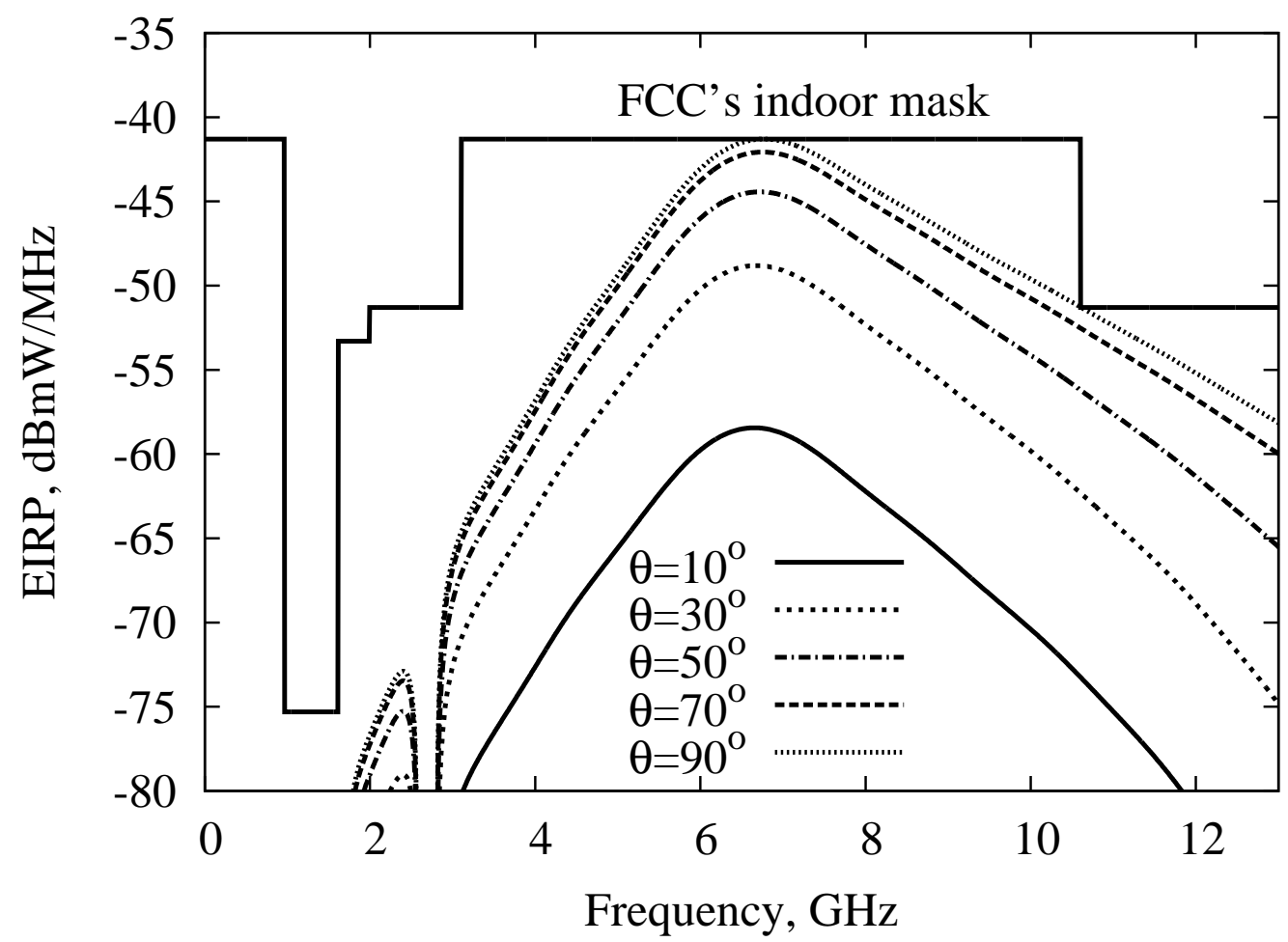

Figure 9: EIRP from the thin-wire antenna

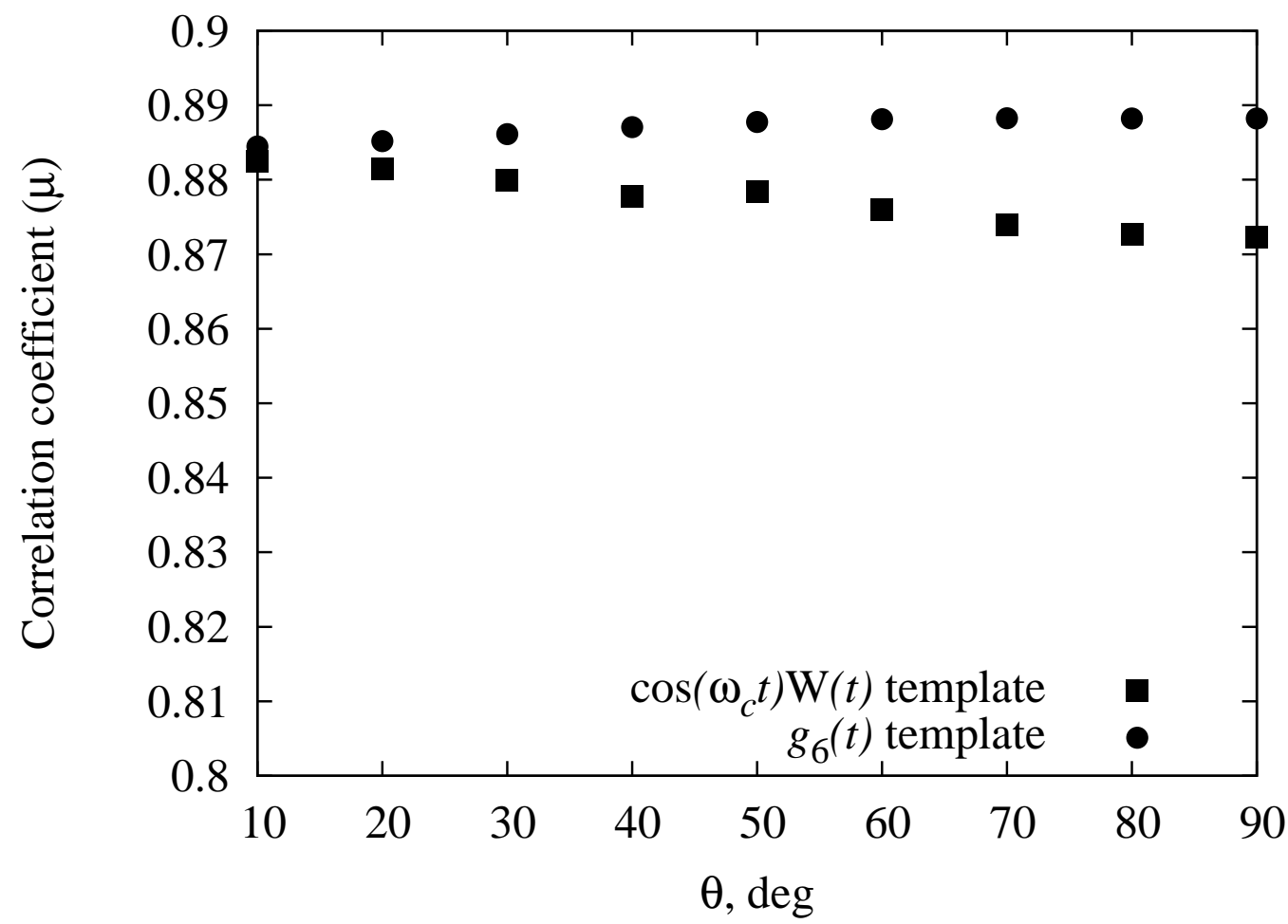

Figure 10: Correlation coefficient of the thin-wire antenna system with different antenna orientations 


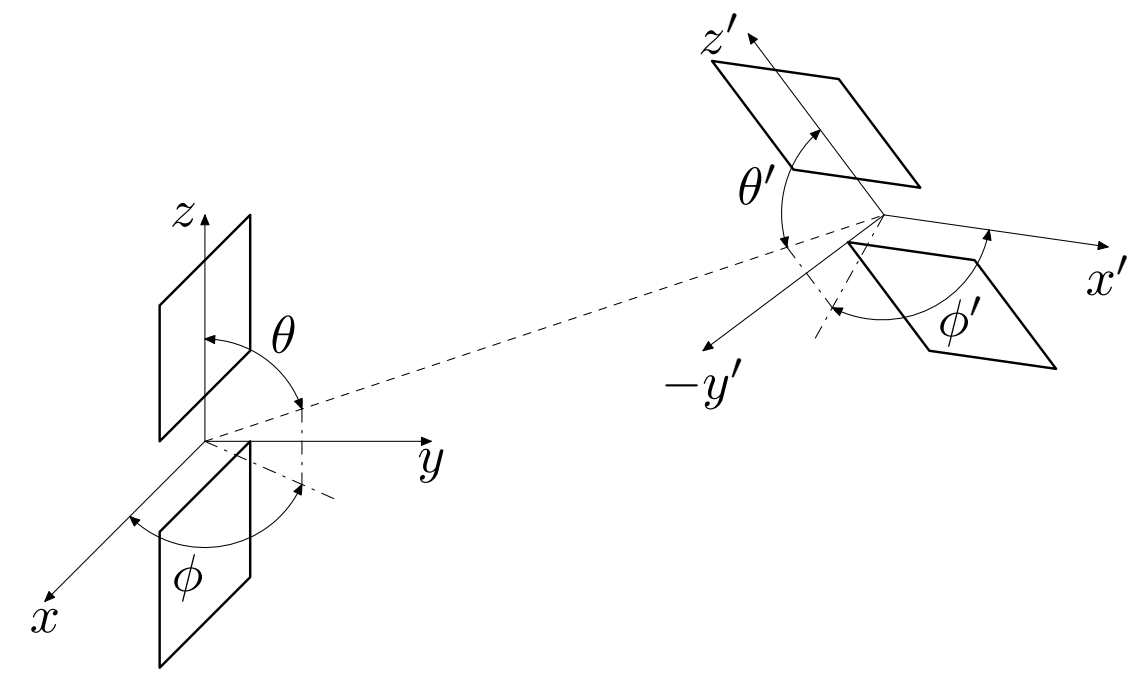

(a) Planar antenna orientations

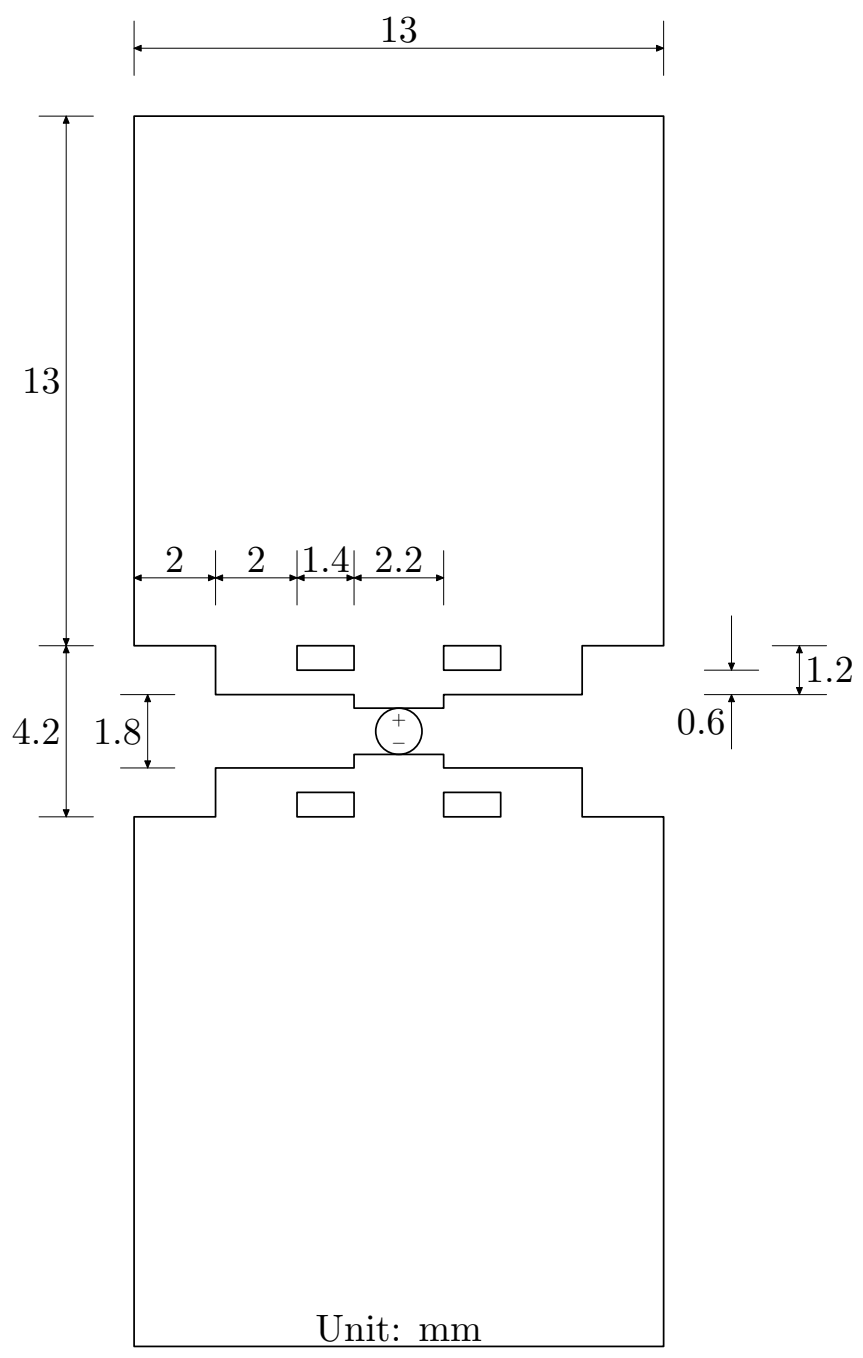

(b) Geometry of the planar dipole antenna

Figure 11: Planar antenna system 


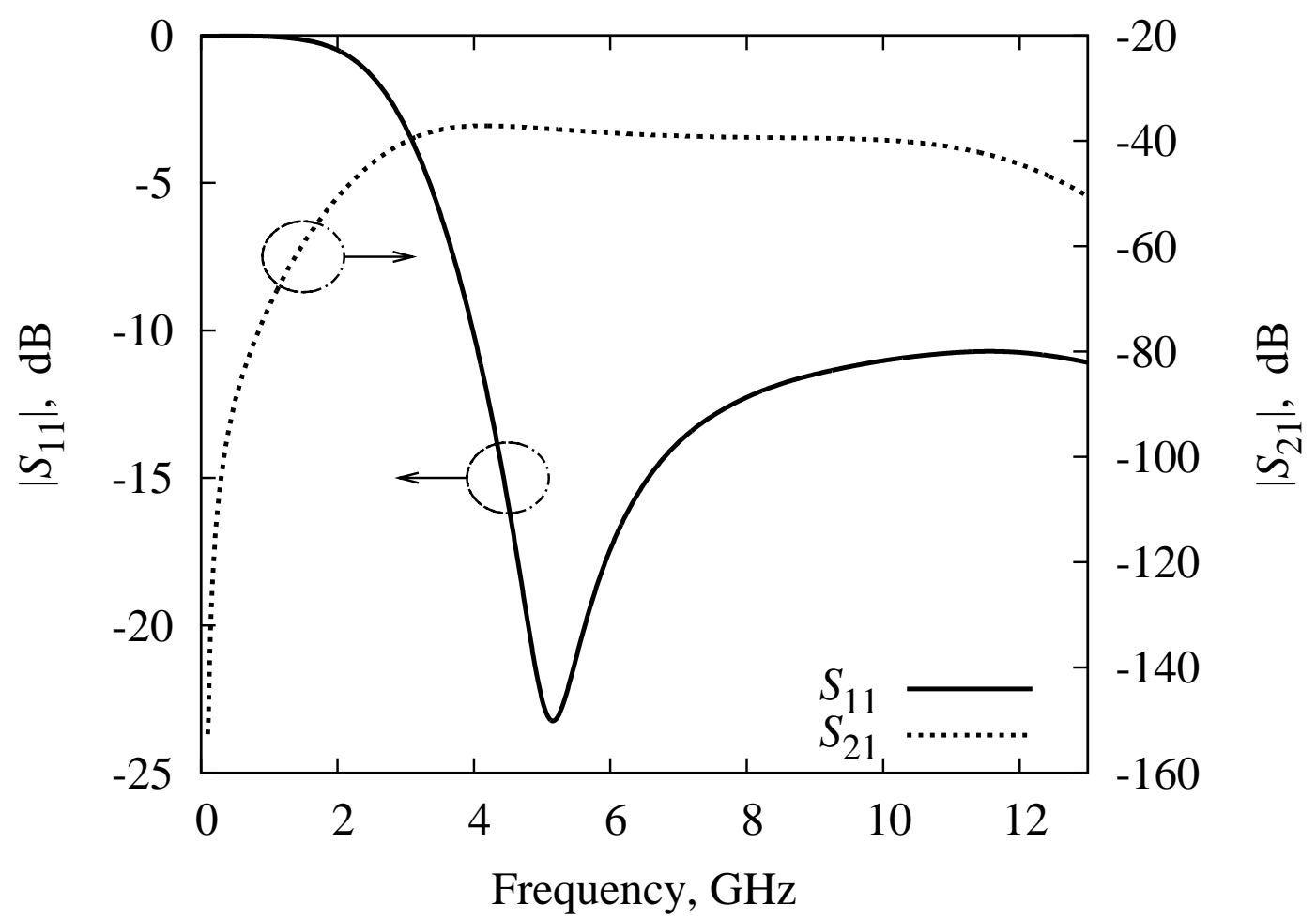

Figure 12: S-parameters of the planar antenna system

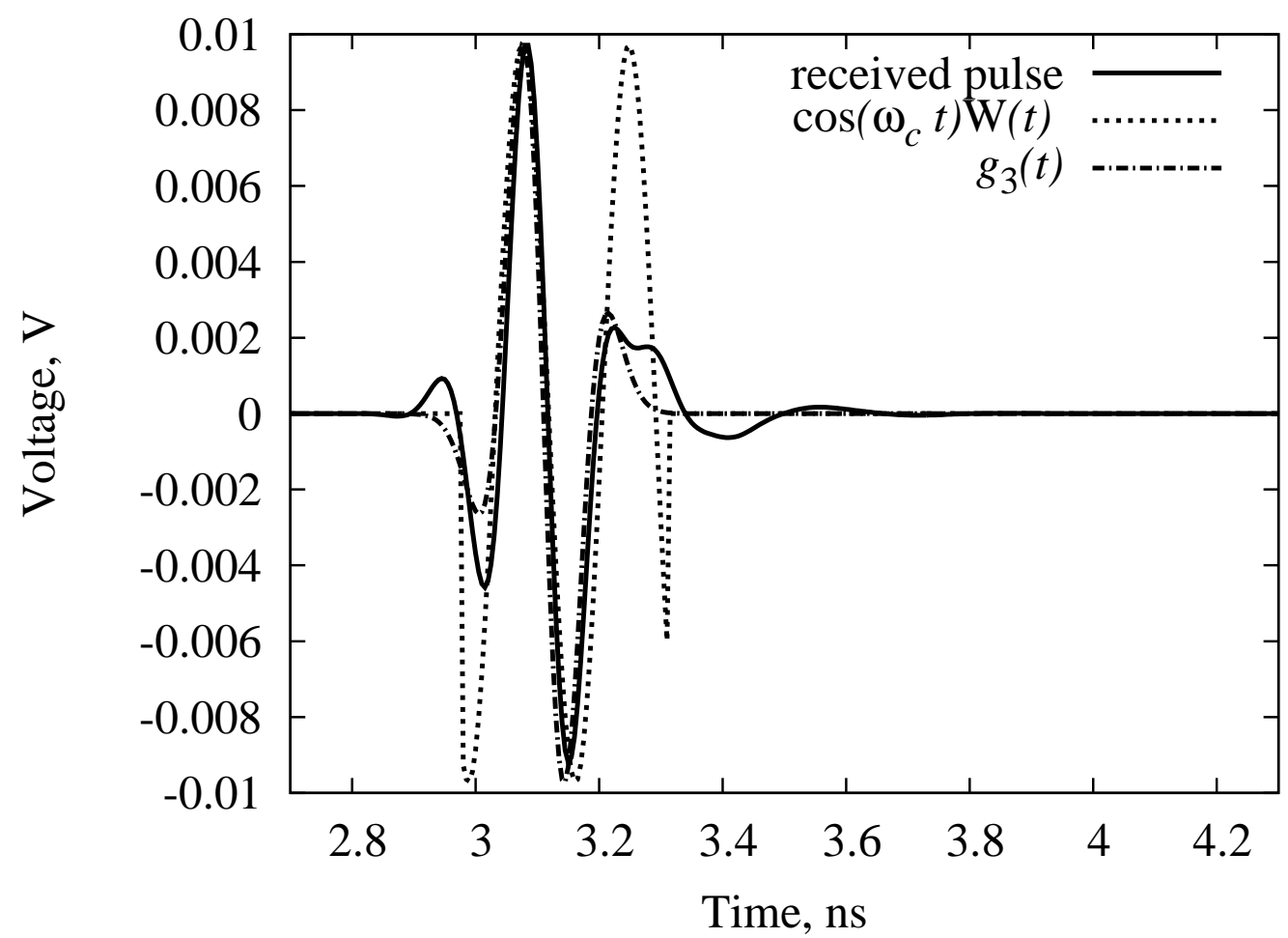

Figure 13: Pulse detection for the planar antenna system 


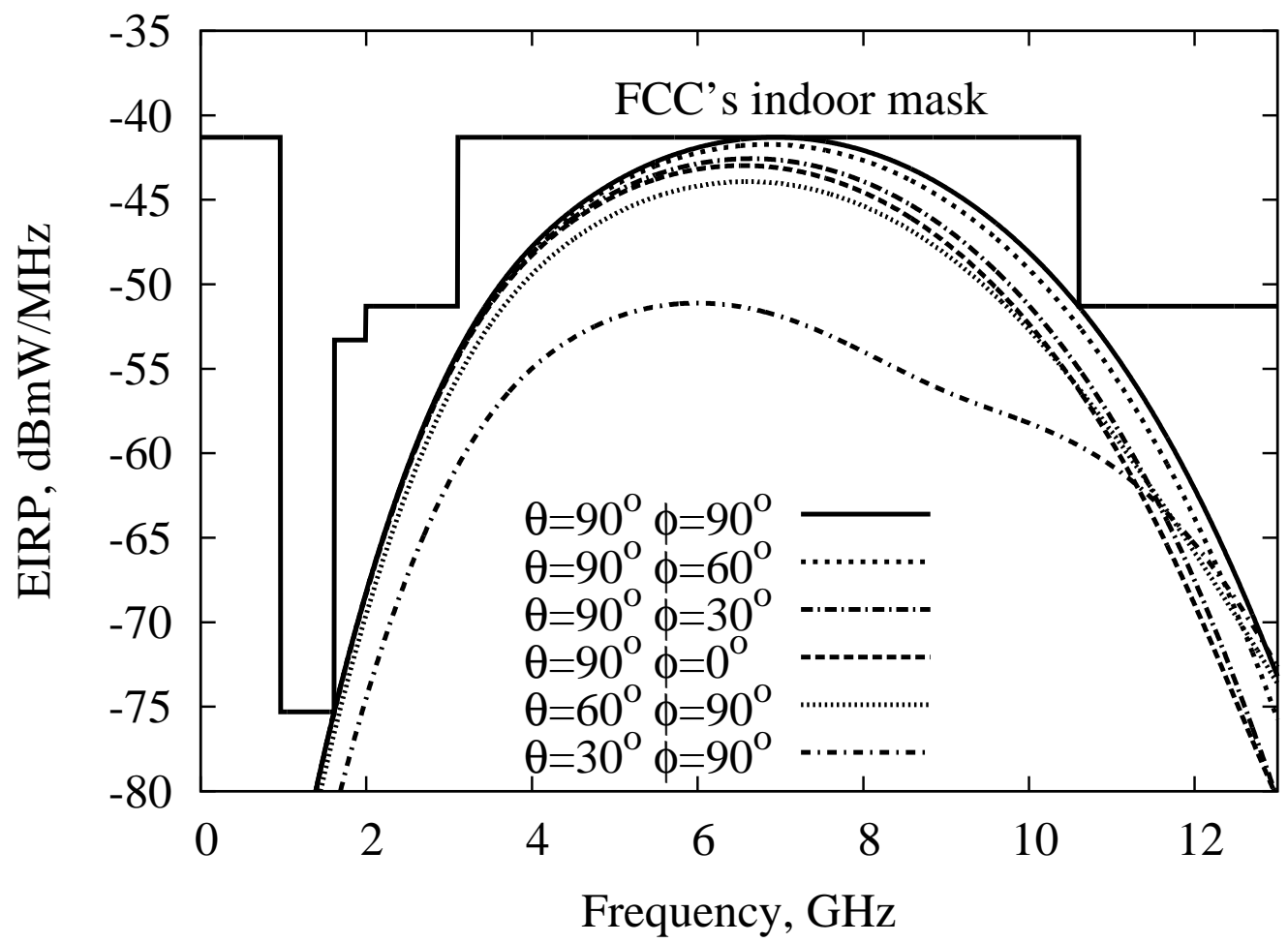

Figure 14: EIRP from the planar antenna

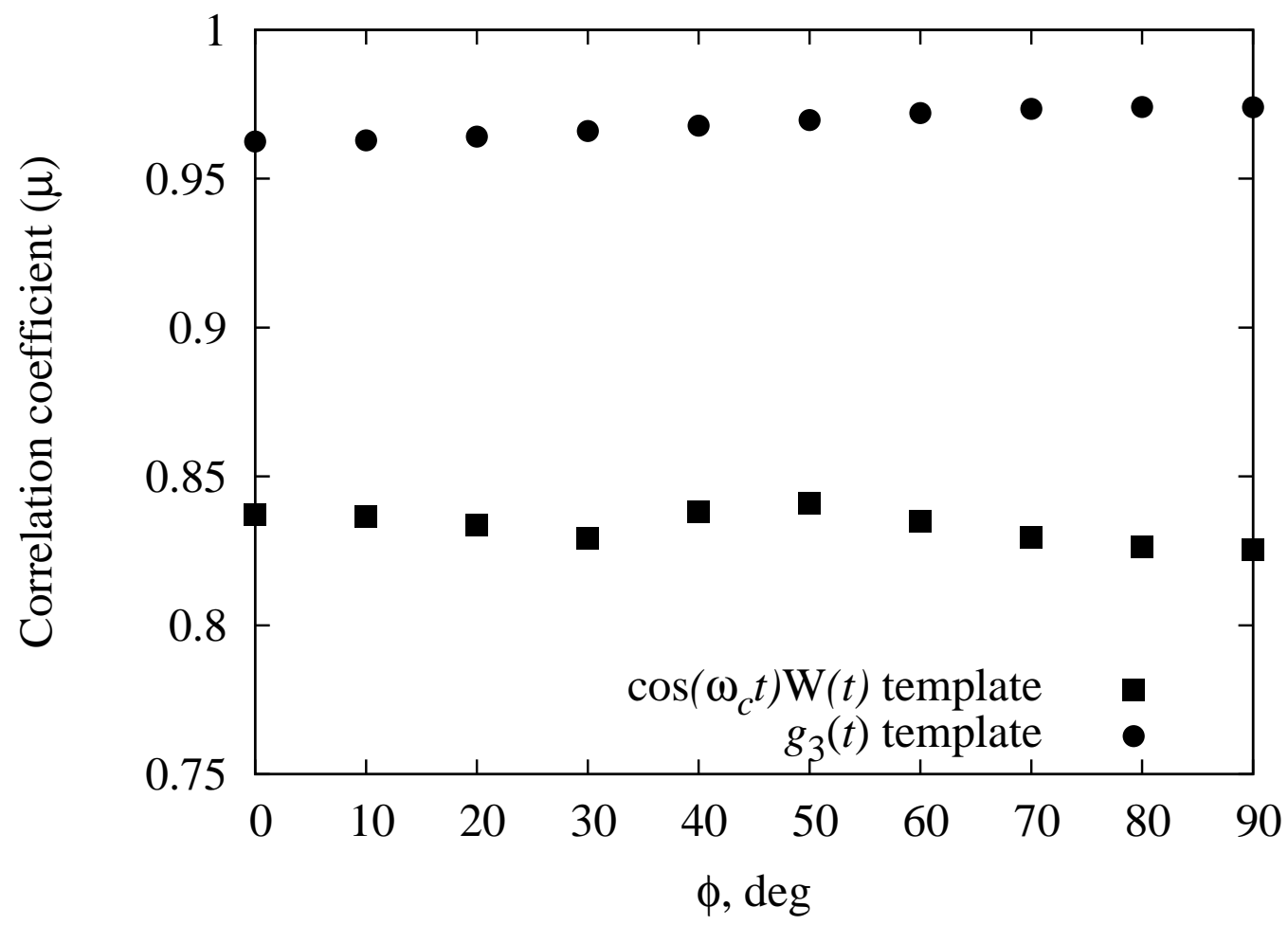

Figure 15: Correlation coefficient of the planar antenna system in the $\theta=90^{\circ}$ plane 


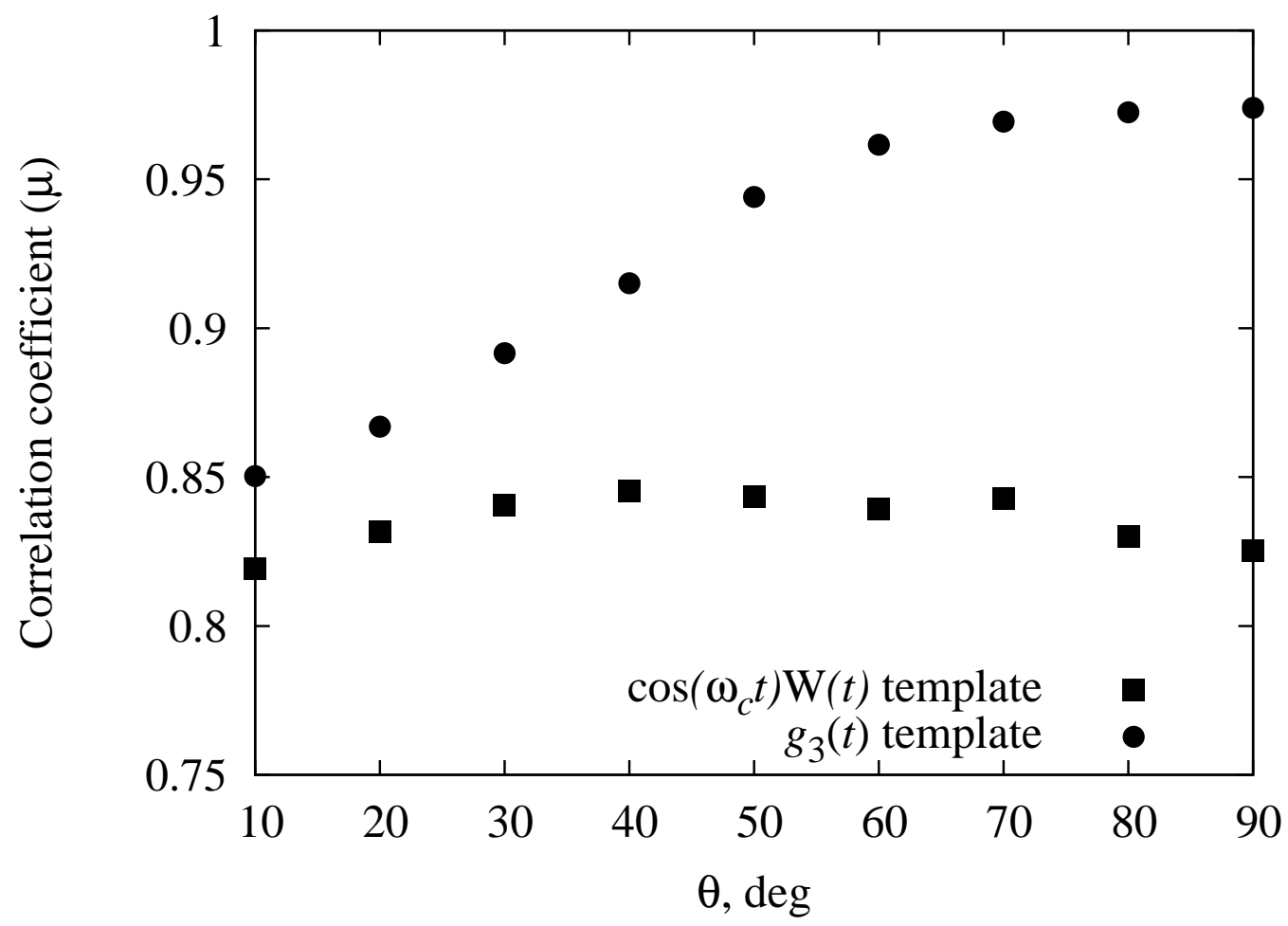

Figure 16: Correlation coefficient of the planar antenna system in the $\phi=90^{\circ}$ plane 
Table 1: Optimized results with the $g_{n}(t)$ template for the thin wire antenna system

\begin{tabular}{cccccc} 
Model & $\sigma_{1}, \mathrm{ps}$ & $\sigma_{2}, \mathrm{ps}$ & $n_{2}$ & $\mu$ & $\eta$ \\
\hline 1 & 40.736 & 81.700 & 6 & $88.9 \%$ & $78.5 \%$ \\
\hline 2 & 40.735 & 81.148 & 6 & $88.8 \%$ & $78.5 \%$ \\
\hline 3 & 40.733 & 81.883 & 6 & $88.9 \%$ & $78.6 \%$ \\
\hline
\end{tabular}

Table 2: Optimized results with the $\cos \left(\omega_{c} t\right) \mathrm{W}(t)$ template for the thin wire antenna system

\begin{tabular}{ccccc} 
Model & $\sigma_{1}, \mathrm{ps}$ & $f_{2}, \mathrm{GHz}$ & $\mu$ & $\eta$ \\
\hline 1 & 40.736 & 6.746 & $87.2 \%$ & $78.5 \%$ \\
\hline 2 & 40.729 & 6.745 & $87.2 \%$ & $78.6 \%$ \\
\hline 3 & 40.731 & 6.740 & $87.2 \%$ & $78.6 \%$ \\
\hline
\end{tabular}

Table 3: Optimized results with the $g_{n}(t)$ template for the planar antenna system

\begin{tabular}{ccccccc} 
Model & $\sigma_{1}, \mathrm{ps}$ & $f_{1}, \mathrm{GHz}$ & $\sigma_{2}, \mathrm{ps}$ & $n_{2}$ & $\mu$ & $\eta$ \\
\hline 1 & 91.602 & 6.655 & 63.191 & 3 & $97.7 \%$ & $95.5 \%$ \\
\hline 2 & 91.428 & 6.651 & 63.188 & 3 & $97.7 \%$ & $95.7 \%$ \\
\hline 3 & 91.792 & 6.628 & 64.993 & 3 & $97.5 \%$ & $95.3 \%$ \\
\hline
\end{tabular}

Table 4: Optimized results with the $\cos \left(\omega_{c} t\right) \mathrm{W}(t)$ template for the planar antenna system

\begin{tabular}{cccccc} 
Model & $\sigma_{1}, \mathrm{ps}$ & $f_{1}, \mathrm{GHz}$ & $f_{2}, \mathrm{GHz}$ & $\mu$ & $\eta$ \\
\hline 1 & 91.531 & 6.661 & 5.769 & $82.7 \%$ & $95.6 \%$ \\
\hline 2 & 91.636 & 6.649 & 5.734 & $82.8 \%$ & $95.5 \%$ \\
\hline 3 & 91.730 & 6.638 & 5.723 & $82.9 \%$ & $95.4 \%$ \\
\hline
\end{tabular}

